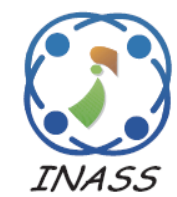

\title{
Fog Removal by Multiple Polynomial Regression Model through Curvelets
}

\author{
Monika Verma $^{1^{*}} \quad$ Vandana Dixit Kaushik $^{1} \quad$ Vinay Pathak $^{1}$ \\ ${ }^{I}$ Computer Science and Engineering Department, Harcourt Butler Technical University, Kanpur, India \\ * Corresponding author's Email: mvmonikaverma261@gmail.com
}

\begin{abstract}
Removal of fog in a Single Image has been a challenging task. Fog causes the visibility to drop thus causing problems for the sensors to capture the scene perfectly. In this work the fog is removed by using multiple polynomial regression model through curvelets. The depth map can be obtained by using the Multiple Polynomial Regression Model and the clear image can be obtained by using the curvelets. Curvelet transform are the multiscale directional transformations which are useful in capturing the relevant information even in the presence of noise. Curvelets have a high directional sensitivity which makes them suitable for representing images even in the presence of noise. If the depth information is extracted then the scene radiance can be easily formulated by using the atmospheric scattering model. In terms of efficiency and experimental results the proposed algorithm works better than the existing approaches.
\end{abstract}

Keywords: Curvelets, Atmospheric scattering model, Regression, Mean square error, Peak signal to noise ratio.

\section{Introduction}

Fog reduces the visibility of the objects and thus becomes a major cause for reduction of quality of images. Fog is formed when there is much of water in the atmosphere in the form of vapor. When the light hits these water droplets they scatter light in all directions and a dense white background is formed. And as a result the photography is also hindered. Under poor atmospheric conditions the images captured are not of good quality. Due to the presence of aerosols in the atmosphere the light reflected from the objects in the scene tend to get deflected before they can reach the camera. Due to the poor visibility of the objects these images lack visual appeal. As a result many applications suffer from the degradations that is cause due to fog. The applications may range from commercial use to underwater photography and aerial photography. Satellite imaging can also be used for the applications of cartography and web mapping, land use planning, archaeology and environmental studies. [1]

The problem to remove fog from a single image is an ill posed problem as the number of unknown variables in the mathematical model is more than the known ones. A number of solutions have been proposed. The atmospheric scattering model is the one widely used. In this model the foggy image is composed of two components which are added together. The first is the scene radiance and second is the airlight component.

In this paper a novel method for removing fog is given which is based on the Multiple Polynomial Regression Model (MPRM) using curvelets. In the present work the scene depth, hue, saturation and value components are calculated by developing a Multiple Polynomial Regression Model and the clarity of images even in the presence of noise is obtained by using curvelets. The brightness and the saturation are the components which have been used in the regression equation. Experimental results show the better performance of the proposed work in comparison with the state of the art methods.

Edge detection is used as a primary method for pattern recognition, image segmentation and scene analysis. An edge detector is a high-pass filter that can be applied for extracting the edge points within an image. Curvelets represent edges better than wavelets. [2] 
In the early researches the defogging used the conventional approach of histogram processing to remove the haze [3]. In polarization based methods two images of the same scene are taken into consideration $[4,5]$.The images taken are with different degrees of polarization. Getting two strictly aligned images is problematic situation. Also they tend to increase the noise and are not beneficial for visibility [6]. The other method that uses multiple images is [7-9]. Here also getting two images that are strictly aligned is a major problem.

In the paper proposed by [10], a parallel image haze remove algorithm is developed, adopting optimized contrast enhancement approach, to optimize the performance based on GPU platform. In [11], the authors have studied the state-of-the-art in the area of fog/haze and have put forward the strengths and weaknesses of various methods.

In the work proposed by [12], the authors have considered the prior based on the local contrast of the image. By using the Markov Random Field, the method maximizes the local contrast of the image. The visibility is enhanced but the image looks oversaturated.

In the dark object subtraction method the assumption is that there must be a dark object in at least one of the three channels [13]. In the proposed algorithm of [14] the authors have introduced Dark Channel Prior (DCP) followed by Weighted Least Square (WLS) and High Dynamic Range (HDR) based fog removal scheme. It has difficulties in removing haze from gray scale image and also the dense haze images cannot be dealt with such an approach. A simple and effective technique of Dark Channel Prior has been proposed in [15]. With the computationally intensive method the sky images are not well handled [15].

The authors of $[16,17]$ have used Markov Random Field to approximate the scene radiance. In [18] the algorithm proposes the regularization dehazing approach to get back the original image. The four types of haze features with Random Forest Transmission are combined together in [19]. The authors in [20] have used the single image color attenuation prior. In spite of the fact that these [1620] methods are showing the remarkable progress, the limitation of the state-of-the-art methods lies in the fact that the haze-relevant priors or heuristic cues used are not effective or efficient enough.

Curvelets are the multiscale transformation that can extract useful information from the images even in the presence of noise. They have directionality property that sets them apart from the rest of the transforms like wavelets, Fourier transforms, Short Term Fourier Transforms.
Digital images always inherit some extent of noise in them. This noise affects the information content of the image. Removal of this noise is very important to extract useful information from an image. However noise cannot be eliminated, it can only be minimized due to overlap between the signal and noise characteristics. [25]

In the present work the Multiple Polynomial Regression Model is used. The model used takes saturation and brightness as the inputs to calculate the depth. This method gives importance to the saturation as well as the brightness. As the fog increases the brightness also increases and the saturation decreases. Hence in the regression model these two parameters are considered. The results are of high quality. In addition the curvelets are also used. Hence the overall performance increases very much. The images are of great clarity. The paper is divided into the following sections. Section 2 describes the fundamental Atmospheric Scattering Model and the curvelets. Section 3 provides the experimental results. Image quality is evaluated by the standard metrics. In section 4 , the conclusions and the future scope are given. In this paper a novel method to remove fog using the Multiple Polynomial Regression Model using Curvelet transformation has been proposed. A comparison has been made with several states of the arts methods.

\section{Methodology}

\subsection{Atmospheric scattering model}

The Atmospheric Scattering Model given by McCartney is used to find out the fog/haze free image [21]. Equation of fog/haze is defined as

$$
\begin{aligned}
& \boldsymbol{H}(\boldsymbol{x})=\boldsymbol{C}(\boldsymbol{x}) t(\boldsymbol{x})+\boldsymbol{A} \boldsymbol{t}_{\boldsymbol{m}}(1-t(\boldsymbol{x})) \\
& t(\boldsymbol{x})=e^{-(s c(d p(x)))}
\end{aligned}
$$

where $\boldsymbol{x}=(x, y)$ is a 2D vector, $\boldsymbol{H}(\boldsymbol{x})$ is a $3 \mathrm{D}$ RGB vector of the color at a pixel(pixel of the hazy image), $\boldsymbol{C}(\boldsymbol{x})$ is a $3 \mathrm{D}$ RGB vector of the color of the light reflected by the scene point at $\boldsymbol{x}$ (pixel of a fog/haze free image). $\boldsymbol{C}$ is known as the scene radiance, $t$ is a map called transmission or transparency of the fog/haze. $t(\boldsymbol{x})$ is a scalar in $[0,1]$, $s c$ represents the scattering coefficient of the atmosphere, $d p(\boldsymbol{x})$ is the depth of a particular pixel. $d p(\boldsymbol{x})$ is assumed to be infinite for pixels that are values of sky regions.

The term $\boldsymbol{C}(\boldsymbol{x}) t(\boldsymbol{x})$ is known as direct attenuation. Attenuation is due to the particles in the atmosphere. The particles are providing hindrance in the way of 
light. They don't allow the entire light to fall on the objects as a result of which the light that is reflected from the objects is less than what they would have reflected when the scene was fog/haze free. Because of direct attenuation the brightness and the contrast of the scene objects decreases in a multiplicative manner. The transmission term is the ratio of light that is reaching the observer without getting attenuated to the light that is reflected by the object. $\boldsymbol{A} \boldsymbol{t}_{\boldsymbol{m}}$ is the atmospheric light. It is a $3 D$ RGB vector assumed to be spatially constant.

The term $\boldsymbol{A} \boldsymbol{t}_{\boldsymbol{m}}(1-t(x))$ is known as the airlight[22]. Due to the Airlight the brightness of the scene increases because in the first place the particles are absorbing the light and then they tend to scatter light in different directions thus becoming the tiny sources of light that keep floating in the atmosphere. The airlight increases in proportion to the amount of fog/haze. So, overall the effect of airlight is more than direct attenuation because of which the brightness of the hazy image is much more and on the other hand the hue and the saturation are having low values.

To solve the above equation the most important information is the depth information of the pixels. Once the depth is found out the transmission becomes an easy parameter to fetch as the term ' $s c^{\prime}$ which is known as the scattering coefficient is assumed to be constant in homogeneous atmospheric conditions. To find out the parameter of airlight we notice that once the transmission is zero then the equation is reduced to Eq. (3).

$$
\text { if } \begin{aligned}
& d p(\boldsymbol{x})->\infty \text { then } \\
& (s c)(d p(\boldsymbol{x}))->\infty \\
& t(\boldsymbol{x})=e^{-\infty}=1 / e^{\infty}=0 \\
& \boldsymbol{H}(\boldsymbol{x})=\boldsymbol{A t}_{\boldsymbol{m}}
\end{aligned}
$$

As shown in the equation 4 transmission parameter becomes zero if the depth of a pixel $(d p(\boldsymbol{x}))$ is infinite.

$$
t(\boldsymbol{x})=e^{-\infty}=1 / e^{-\infty}=0
$$

Equation 4 says that the transparency of fog/haze is zero if the pixel is quite distant from the camera, i.e., if the value of $t(x)$ is closer to zero then more haze is there. Geometrically the fog/haze equation describes the transmission $t$ as the ratio of the two line segments.

$$
t(x)=\left\|A t_{m}-H(x)\right\| /\left\|A t_{m}-C(x)\right\|
$$

In RGB color model the vectors $\boldsymbol{A}, \boldsymbol{I}(\boldsymbol{x})$ and $\boldsymbol{J}(\boldsymbol{x})$ are coplanar and their corresponding end points are collinear.

To solve the Atmospheric Scattering Model the parameter of depth is found by using the Multiple Polynomial Regression model (MPRM). ' $\mathrm{sc}^{\prime}$ is assumed to be constant in homogeneous atmospheric conditions.

Let $D$ be the depth of the image for which the coefficients are to be calculated. Using the FRIDA data sets the images with their corresponding depth maps are used (if known). Once the coefficients are known then they can be applied in general to any of the images. The depth estimation $\left(D_{E}\right)$ is done by using the Multiple Polynomial Regression Model. The approach followed is mathematically explained below:

$$
D_{E}=\alpha_{0}+\alpha_{1}(s(\boldsymbol{x}))+\alpha_{2}\left(b^{2}(\boldsymbol{x})\right)+\operatorname{err}(\boldsymbol{x})
$$

where $\alpha_{0}, \alpha_{1}, \alpha_{2}$ are the coefficients, $b$ is the brightness component of the image, $s$ is the saturation value of the image and err is the error in the model. The err is the random error of the model which is be assumed to be negligible. The model is linear as it is linear in the parameters $\alpha_{0}, \alpha_{1}$ and $\alpha_{2}$. This model describes a three dimensional space in $D$, $b^{2}(x), s(x)$. The coefficient $\alpha_{0}$ is the intercept of this plane. The parameters $\alpha_{1}, \alpha_{2}$ are known as the partial regression coefficients. Parameter $\alpha_{1}$ represents the change in the mean response corresponding to a unit change in $s(x)$ provided that $b^{2}(x)$ is constant.

In the same way the parameter $\alpha_{2}$ represents the change in the mean response corresponding to a unit change in $\mathrm{b}^{2}(\mathrm{x})$ provided that $\mathrm{s}(\mathrm{x})$ is constant. Multiple Polynomial Regression Models (MPRM) contains squared and higher order terms of the predictor variables making the response surface curvilinear.

$$
\begin{gathered}
d_{1}=\alpha_{0}+\alpha_{1} s\left(x_{11}\right)+\alpha_{2} b^{2}\left(x_{12}\right)+\operatorname{err}\left(x_{1}\right) \\
d_{2}=\alpha_{0}+\alpha_{1} s\left(x_{21}\right)+\alpha_{2} b^{2}\left(x_{22}\right)+\operatorname{err}\left(x_{2}\right) \\
\cdot \\
\cdot \\
\cdot \\
d_{i}=\alpha_{0}+\alpha_{1} s\left(x_{i 1}\right)+\alpha_{2} b^{2}\left(x_{i 2}\right)+\operatorname{err}\left(x_{i}\right) \\
\cdot \\
\cdot \\
\cdot \\
d_{n}=\alpha_{0}+\alpha_{1} s\left(x_{n 1}\right)+\alpha_{2} b^{2}\left(x_{n 2}\right)+\operatorname{err}\left(x_{n}\right)
\end{gathered}
$$

The system of $n$ equations shown previously can be represented in matrix notation as follows:

$$
D=X \alpha+e r r
$$


$\mathbf{D}, \mathbf{X}, \boldsymbol{\alpha}$, err are all vectors. The elements of these vectors are defined below:

where $\begin{aligned} D & =\left[\begin{array}{c}d_{1} \\ d_{2} \\ d_{3} \\ \cdot \\ \cdot \\ \cdot \\ d_{n}\end{array}\right] & X=\left[\begin{array}{ccc}1 & x_{11} & x_{12} \\ 1 & x_{21} & x_{22} \\ 1 & x_{31} & x_{32} \\ & \cdot & \\ & \cdot & \\ 1 & x_{n 1} & x_{n 2}\end{array}\right] \\ & \alpha=\left[\begin{array}{c}\alpha_{0} \\ \alpha_{1} \\ \alpha_{2}\end{array}\right] & \operatorname{err}=\left[\begin{array}{c}e r r_{1} \\ e r r_{3} \\ \cdot \\ \cdot \\ \cdot \\ e r r_{n}\end{array}\right]\end{aligned}$

The matrix $X$ is known as the design matrix. The vector $\boldsymbol{\alpha}$ contains the regression coefficients. To obtain the regression model, $\boldsymbol{\alpha}$ should be known. $\boldsymbol{\alpha}$ is estimated using least square estimates. The following equation is used:

$$
\boldsymbol{\alpha}=\left(X^{\prime} X\right)^{-1} X^{\prime} D
$$

After reading the image which is degraded due to haze, the RGB color model of the hazy image is converted to the hue, saturation and value color model. After extracting the brightness component of the hazy image, it is histogram equalized. The rest of the processing is done on the histogram equalized image. The depth of the image is calculated by using the Multiple Polynomial Regression Model.

If the depth map is given (e.g. from the FRIDA data set) then the values of the coefficients $\alpha_{0}, \alpha_{1}, \alpha_{2}$ are found by solving the equation (7). If the depth map is not given then it is artificially generated. For every image that is free from haze a random depth map is generated. It should be of the same size as the image. A standard uniform distribution is used on the interval $(0,1)$. With the help of synthetic depth map the values of the coefficients $\alpha_{0}, \alpha_{1}$ and $\alpha_{2}$ are found out. Finally a hazy image is generated by using the equation (1) and equation (2). Once the values of these parameters are known then the scene radiance can be found out by using the equation given below:

$$
C(x)=\left[H(x)-A t_{m}(1-t(x))\right] / t(x)
$$

All printed material, including text, illustrations, and charts, must be kept within a print area of 17.2 $\mathrm{cm}$ wide by $24.62 \mathrm{~cm}$ high. For A4 paper, all the pages should begin $2.54 \mathrm{~cm}$ from both the top and the bottom edge, and $1.9 \mathrm{~cm}$ both from the left and the right edge. The page header is expected to be 1.5 $\mathrm{cm}$ high, and the page footer should be height of $1.75 \mathrm{~cm}$. Do not write or print anything outside the print area. All text must be in a two-column format. Columns are to be 22.2 times of the character size, with a space size of 2.02 times of the character size. Text must be fully justified.

A format sheet with the margins and placement guides is available as both Word and PDF files as $<$ format.docx $>$ and <format.pdf>. It contains lines and boxes showing the margins and print areas. If you hold it and your printed page up to the light, you can easily check your margins to see if your print area fits within the space allowed.

\subsection{Curvelets}

Curvelets are mathematical transformations which are multiscale directional transforms. Their elements are identified by the scale and location parameters. They have a very high degree of directional specificity [23]. They are better than wavelets in terms of handling of discontinuities. While many wavelets coefficients are needed for the representation of the curves in the images, a few curvelet coefficients are required for representing them. They are also suitable to detect the relevant information even in the presence of noise in which the wavelets have less efficiency. Curvelets allow an almost optimal nonadaptive sparse representation of the object with edges. The new ridgelet and curvelet transforms were developed to overcome the limitations of wavelets. Wavelet transform of images exhibits large wavelet coefficients even at fine scales, all along the important edges in the image, so that in a map of the large wavelet coefficients one sees the edges of the images repeated at scale after scale. With many coefficients to estimate, denoising faces certain difficulties. [26].

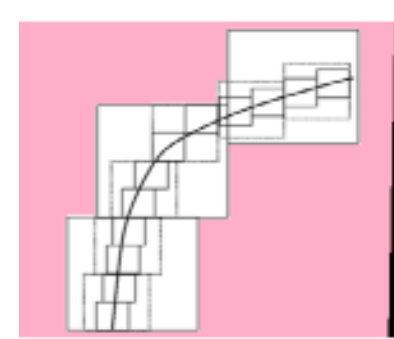

(a)

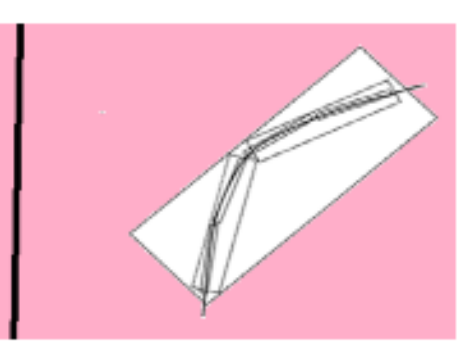

(b)
Figure.1 Comparison between wavelets and curvelets on account of edges in the image: (a) Many wavelet coefficients and (b) Few curvelet coefficients 


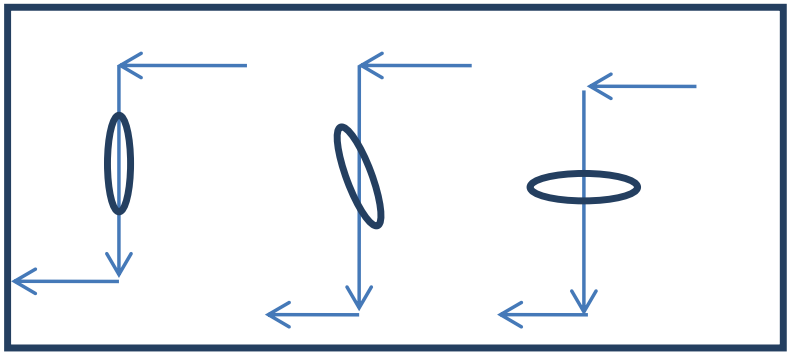

Figure.2 Curvelets at different orientations, with fixed scale and location

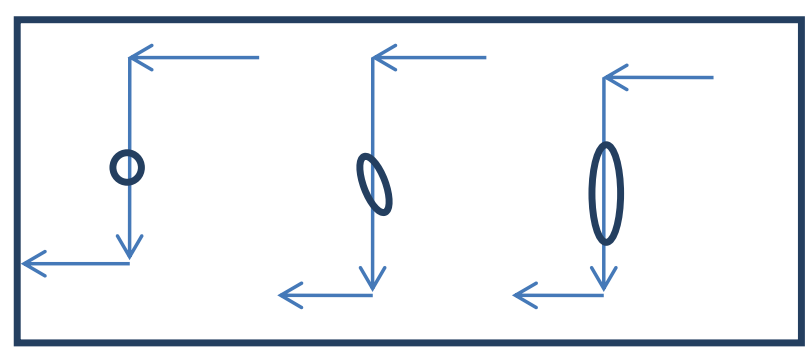

Figure.3 Curvelets at different scales, with fixed orientation and location

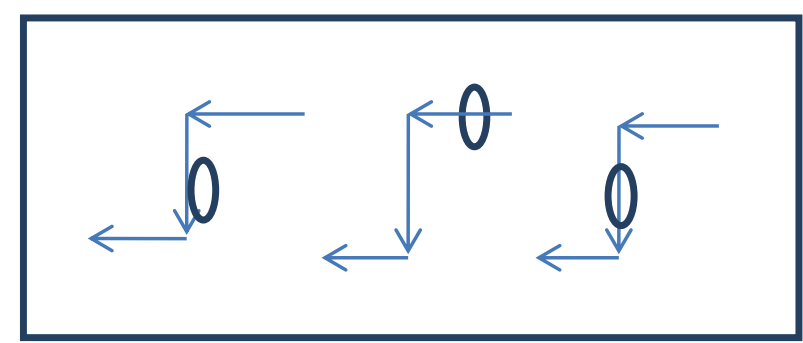

Figure.4 Curvelets at different locations, with fixed scale and orientation

The curvelet transformation represents edges better than wavelets. Edges play a fundamental role in image understanding so edge enhancement becomes a good way to enhance the spatial resolution. Curvelets provide rich information content both spectrally as well as spatially. In images the two dimensional discontinuities are present which curvelets can be of great use. Curvelets are basically comprised of three main functions
A) Sub band decomposition
B) Smooth partitioning
C) Ridgelet analysis

Curvelets are based on multiscale ridgelets which are a combination of radons and wavelets. In other words a curvelet is a multiscale ridgelet which lives in a particular frequency band. The band pass is set so that the curvelet length and width at fine scales are related by a scaling law (width $=$ length $^{2}$ ). It allows curvelets to act like needle in the fine scales, hence the anisotropy increases with decreasing scales like a power law.

In Fig. 2, the curvelets are given at various orientations. Looking at the figure one can notice that the curvelets energy is maximum in the first part as the curvelet is covering the entire edge. The second part is representing a curvelet which does not cover the entire edge so the curvelet coefficients are having less value than the first case. In the third part the curvelet is consisting of very less edge energy.

They are suitable for having stable, near optimal representation of the smooth objects having discontinuities along curves in an efficient way. Curvelets use very few coefficients for a given accuracy of reconstruction. They are designed to represent images on a piecewise smooth curves very sparsely. The features of curvelet transform are different from the other transforms in a way that in case of other transforms like wavelets, multiwavelets, steerable pyramids the frame elements don't have high directional sensitivity and neither are highly anisotropic.

In Fig. 3, given below, the curvelets are given at various scales. The curvelets energy is at maximum in the third part as the curvelet is covering the entire edge. The curvelet is acting as a needle at fine scales. The second part is representing a curvelet which is slightly oval and it does not cover the entire edge so the curvelet coefficients are having less value than the third case. For a coarse scale i.e., the first part the curvelet is consisting of a large area without edge energy.

In Fig. 4 the curvelets are given at various locations. Looking at the figure one can notice that the curvelets energy is maximum in the first part as the curvelet is not at all covering the edge. So the curvelet coefficients become zero in the first case. The second part is representing a curvelet which does not cover the entire edge so the curvelet coefficients are having slightly more value than the first case. In the third part the curvelet is consisting of maximum edge energy. [24].

An algorithmic approach to define the steps clearly is given below:

\section{Algorithm 1.}

1.Take the Input hazy image.

2.Find out the histogram equalized "value" component of the hazy image after converting it to HSV color model.

3.The rest of the processing is done on the image acquired by step 2 .

4.Calculate the depth of the image, by the following equation: $D_{E}=\alpha_{0}+\alpha_{1}(s(\boldsymbol{x}))+\alpha_{2}$ $\left(b^{2}(\boldsymbol{x})\right)+\operatorname{err}(\boldsymbol{x})$ where the parameters $b(x)$ and $s(x)$ are the brightness and the saturation channels of the unclear image. $\alpha_{0}, \alpha_{1}, \alpha_{2}$ are the coefficients whose values come out to be: .91, $.64,-.28$. 
5.Once the depth is found the transmission is calculated by equation number: 2 .

6. When the transmission is known then the value of clear image is found out by equation number: 11.

7.Once the image is obtained apply curvelets over the image.
I. Sub band decomposition
II. Smooth partition
III. Ridgelet analysis

\section{Experimental results}

Given below in figure 5 are the unclear images of fog/haze. The data set used is FRIDA Data Sets $[24,25]$. Many real images are also used for the experimental results. More than 1000 images have been tested for the same. This paper has considered two types of images a) fog/haze synthetic images and b) natural images.

The rows corresponding to Figure 6 denotes the results generated from the various algorithms. The first row corresponds to algorithm of $\mathrm{He}$ et al; second row corresponds to Qingsong et al, third to the proposed MPRM and the fourth to the MPRM with curvelets.

In the algorithm proposed by $\mathrm{He}$ et al., (first row) the blocking artifacts are there. In addition to the blocking artifacts the objects which are far away from the camera are not seen beyond a particular distance. The images in the third row are better than the existing algorithms in terms of the SSIM, L2rat, Mean Square Error, Peak Signal to Noise Ratio. The fourth row corresponds to the results obtained after applying the curvelets also to the MPRM. The best results are corresponding to the fourth row. More than thousand images are compared. The assessment is done by using these four parameters given below:

\subsection{L2RAT}

L2RAT is the ratio of the squared norm of the signal or image approximation, XAPP, to the input signal or image, $X$. Squared norm of the signal or L2-norm is also known as least squares. It is essentially minimizing the sum of the square of the differences (SD) between the target value $\left(\mathbf{Z}_{\mathbf{i}}\right)$ and the estimated values $\mathbf{f}\left(\mathbf{x}_{\mathbf{i}}\right)$ :

$$
S D=\sum_{i=1}^{n}\left(z_{i}-f(x)_{i}\right)^{2}
$$

The table given below compares the results of various methods applied on foggy images in terms of L2RAT. The images 1-5 are shown in figure 5. Figure7 shows the comparison of sixty images on the L2RAT.

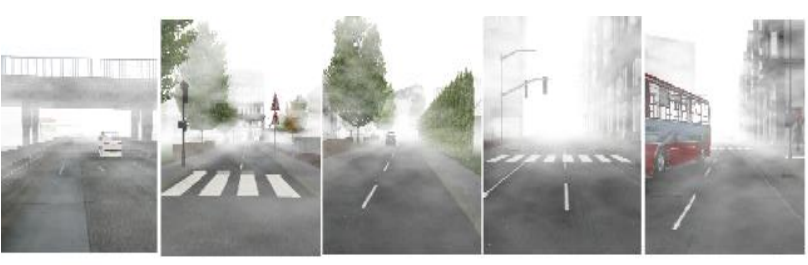

Figure.5 The figure is representing the heterogeneous images

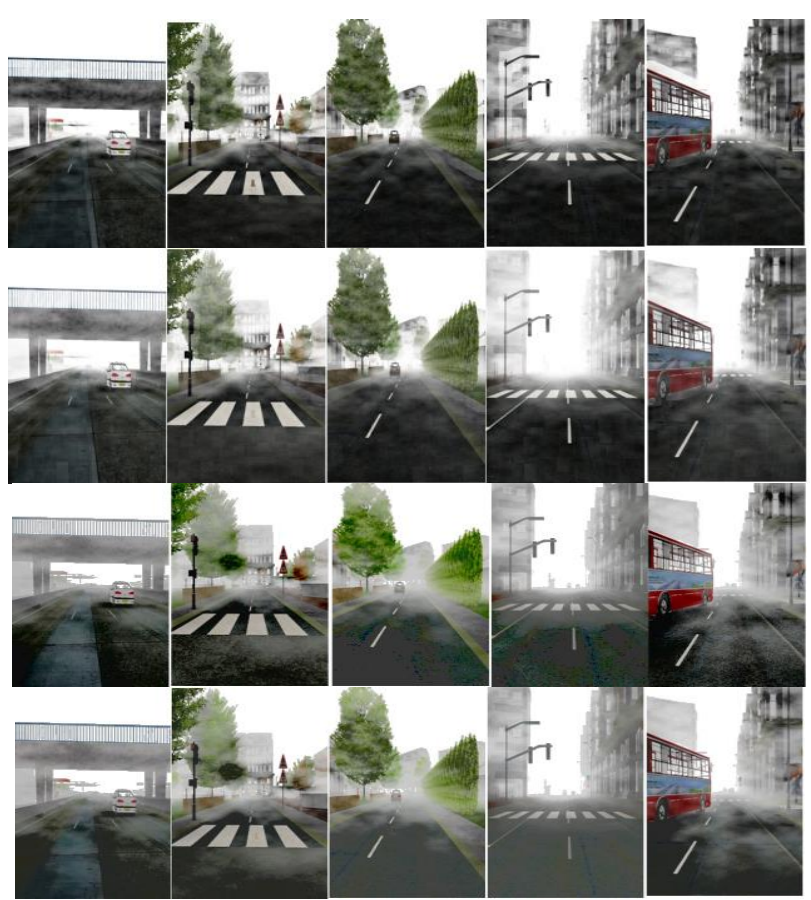

Figure. 6 Images obtained after applying the algorithms of He qt al [15], Qingsong et al [20], Multiple polynomial

Regression Model, MPRM using curvelets

Table 1. Comparison of the various algorithm on the basis of L2RAT of He et al. [15], Qingsong et al. [20]

\begin{tabular}{|c|c|c|c|c|c|}
\hline Images & $\mathbf{1}$ & $\mathbf{2}$ & $\mathbf{3}$ & $\mathbf{4}$ & $\mathbf{5}$ \\
\hline He et. al & 1.14 & 1.04 & 1.05 & 1.04 & .92 \\
\hline $\begin{array}{c}\text { Qingsong } \\
\text { et al. }\end{array}$ & 1.12 & 1.08 & 1.10 & 1.06 & 1.05 \\
\hline MPRM & 1.05 & .9 & 1.00 & .96 & 1.00 \\
\hline Proposed & 1.00 & .96 & 1.00 & .97 & 1.00 \\
\hline
\end{tabular}

\subsection{Mean square error}

If comparison of two images is made based on their respective qualities then a metrics called Mean Square Error is used. The MSE between two images $g(x, y)$ and $f(x, y)$ is

$$
e_{M S E}=1 / M N \sum_{n=1}^{M} \sum_{m=1}^{N}[f(n, m)-g(n, m)]^{2}
$$

where $M$ and $N$ represent the number of rows and columns respectively. The table given below compares the results of various methods applied on foggy images in terms of Mean Square Error. The 


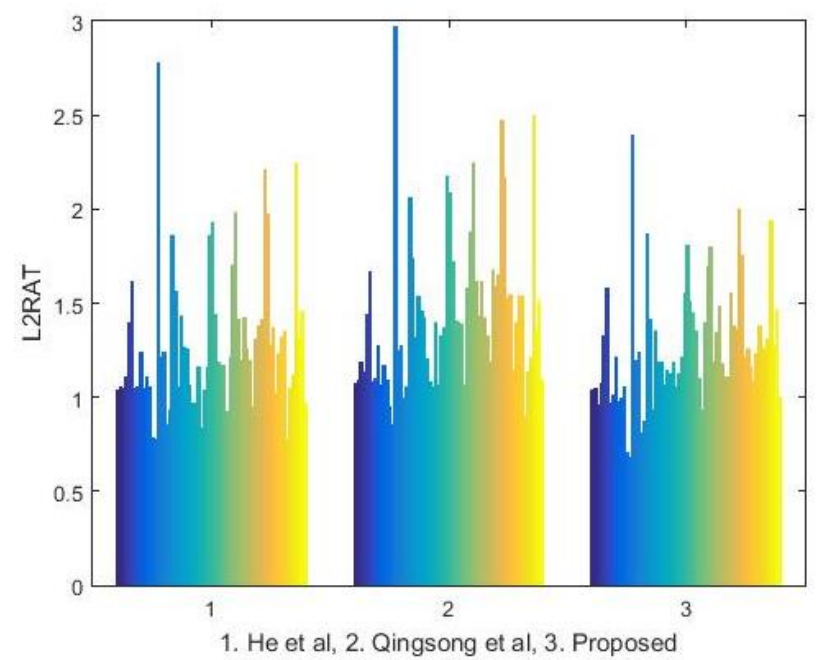

Figure.7 On the $\mathrm{X}$ axis the three approaches are given. 1 He et al, 2. Qingsong et al, and 3.Proposed. Comparison is made on the basis of L2RAT

Table 2. Comparison of the various algorithm on the basis of MSE of He et al. [15], Qingsong et al. [20]

\begin{tabular}{|l|c|c|c|c|c|}
\hline \multicolumn{1}{|c|}{ Images } & $\mathbf{1}$ & $\mathbf{2}$ & $\mathbf{3}$ & $\mathbf{4}$ & $\mathbf{5}$ \\
\hline He et. al & 2805 & 2624 & 2199 & 2361 & 4360 \\
\hline $\begin{array}{l}\text { Qingsong } \\
\text { et al. }\end{array}$ & 2597 & 2482 & 2168 & 2356 & 3916 \\
\hline MPRM & 2022 & 1982 & 1555 & 1136 & 3364 \\
\hline Proposed & 1396 & 1659 & 1234 & 921 & 2939 \\
\hline
\end{tabular}

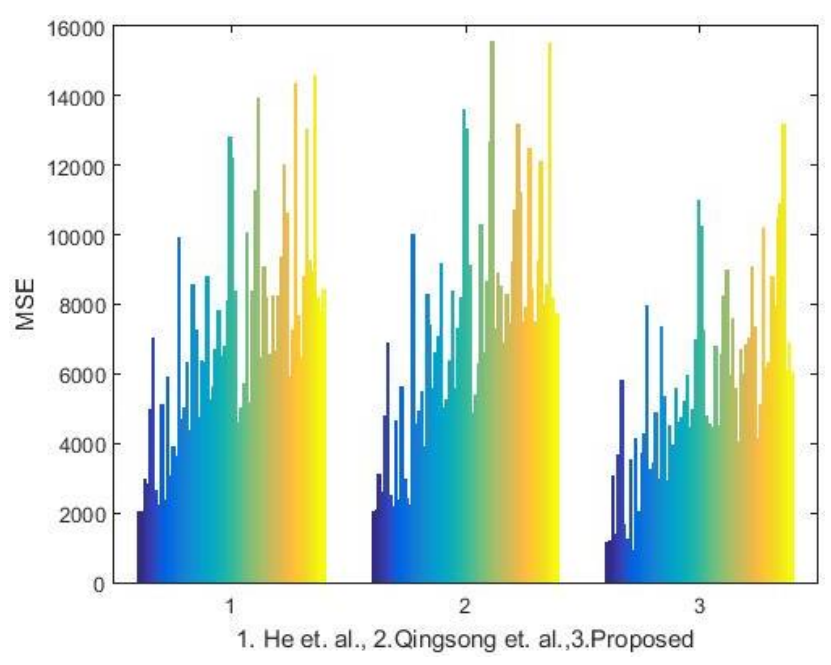

Figure. 8 On the $\mathrm{X}$ axis the three approaches are given. 1 He et al., 2. Qingsong et al., and 3. Proposed. Comparison is made on the basis of MSE

images 1-5 are shown in figure5. Figure 8 shows the comparison of sixty images on the MSE.

\subsection{Peak signal to noise ratio}

PSNR is the ratio between the maximum possible power of a signal and the power of corrupting noise that affects the fidelity of its representation. Because many signals have a very
Table 3. Comparison of the various algorithm on the basis of PSNR of He et al. [15], Qingsong et al. [20]

\begin{tabular}{|l|c|c|c|c|c|}
\hline \multicolumn{1}{|c|}{ Images } & $\mathbf{1}$ & $\mathbf{2}$ & $\mathbf{3}$ & $\mathbf{4}$ & $\mathbf{5}$ \\
\hline He et. al & 13.65 & 13.94 & 14.70 & 14.39 & 11.73 \\
\hline $\begin{array}{l}\text { Qingsong } \\
\text { et al. }\end{array}$ & 13.98 & 14.98 & 14.76 & 14.40 & 12.20 \\
\hline MPRM & 15.07 & 15.15 & 16.21 & 17.57 & 12.86 \\
\hline Proposed & 16.65 & 15.93 & 17.21 & 18.48 & 13.44 \\
\hline
\end{tabular}

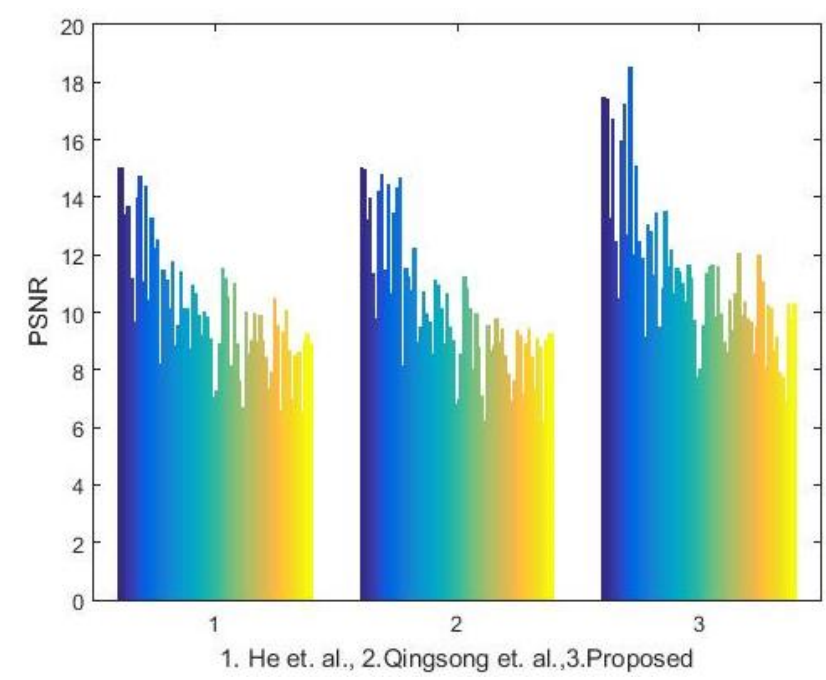

Figure. 9 On the $\mathrm{X}$ axis the three approaches are given. 1 He et al., 2. Qingsong et al., 3. Proposed. Comparison is made on the basis of PSNR

wide dynamic range, PSNR is usually expressed in terms of the logarithmic decibel scale. The PSNR function implements the following equation to calculate the Peak Signal-to-Noise Ratio (PSNR):

$$
\mathrm{PSNR}=-10 \log _{10} \frac{\text { Peak value }^{2}}{M S E}
$$

where peak value is either specified by the user or taken from the range of the image data type $M S E$ is the mean square error, i.e. $M S E$ between A and ref. The table given below compares the results of various methods applied on foggy images in terms of PSNR. Figure 9 shows the comparison of sixty images on the PSNR.

\subsection{Structural similarity index}

The Structural Similarity (SSIM) index is a method for measuring the similarity between two images. The SSIM index can be viewed as a quality measure of one of the images being compared, provided the other image is regarded as of perfect quality. The table given above compares the results of various methods applied on foggy images in terms of SSIM. Fig. 10 shows the comparison of sixty images on the SSIM. 
Table 4: Comparison of the various algorithm on the basis of SSIM of He et al. [15], Qingsong et al. [20]

\begin{tabular}{|c|c|c|c|c|c|}
\hline Images & $\mathbf{1}$ & $\mathbf{2}$ & $\mathbf{3}$ & $\mathbf{4}$ & $\mathbf{5}$ \\
\hline He et. al & .5349 & .5540 & .597 & .568 & .397 \\
\hline $\begin{array}{c}\text { Qingsong } \\
\text { et al. }\end{array}$ & .5725 & .606 & .6432 & .588 & .449 \\
\hline MPRM & .5157 & .5361 & .6126 & .619 & .424 \\
\hline Proposed & .6326 & .6073 & .6605 & .6666 & .4980 \\
\hline
\end{tabular}

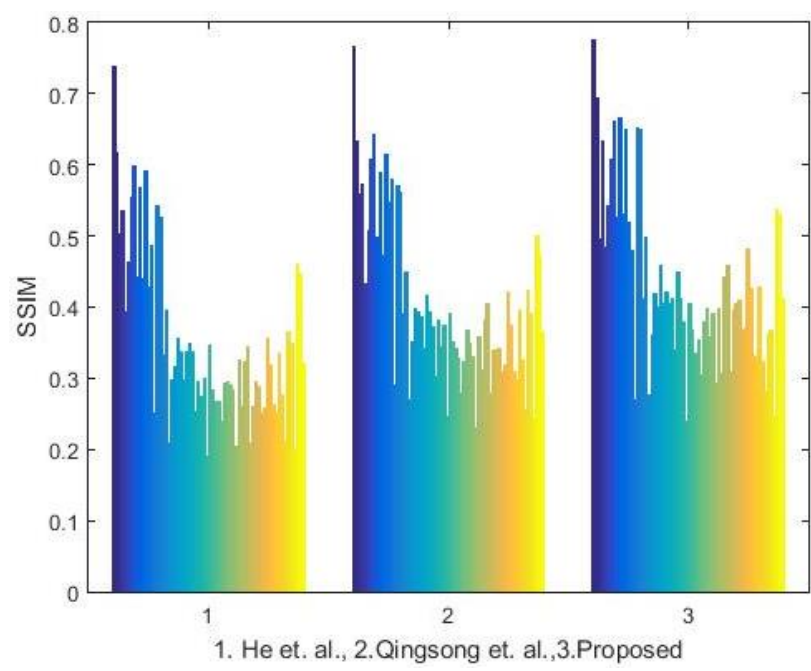

Figure.10 On the $\mathrm{X}$ axis the three approaches are given. 1 He et al., 2. Qingsong et al., and 3. Proposed. Comparison is made on the basis of SSIM

\section{Conclusion and future scope}

In the current work, a novel approach for removing fog is used. Multiple Polynomial Regression Model (MPRM) together with Curvelets is used for the estimation of depth, intensity and saturation of the foggy images. After converting the input foggy image from the RGB to the HSV space, the proposed algorithm takes the histogram equalized values of the 'V'(value) component. The depth is found out by Multiple Polynomial Regression Model. $\alpha_{0}, \alpha 1, \alpha 2$ are the coefficients required in the model whose values come out to be: .91, -.64, -.28.As the fog increases the brightness increases in proportion. Keeping this prior the proposed approach takes the brightness as a squared component. The HSV space is now converted to RGB space again, to get a clear image. The image obtained after obtaining depth is processed through curvelet transformations. The curvelet transformation is a mathematical transform which is having high directional sensitivity. The experimental results show better performance of the proposed approach than the results given by He et al. [15] and Qingsong et al. [20].

The scattering coefficient is assumed to be constant as the atmospheric conditions are taken to be homogeneous. The method would have more flexibility if the than the current approach if the atmospheric conditions are taken into consideration. The resultant images are more towards the gray scale also. So, the future scope of the paper is to work on the concerned issues.

\section{References}

[1] R. Fattal, "Single Image Dehazing", In: Proc. of the ACM Transactions on Graphics, Vol. 27, No. 3, p. 72, 2008.

[2] M. Elhabiby, A. Elsharkawy, N. El-Sheimy, "Second generation curvelet transforms Vs Wavelet transforms and Canny edge detector for edge detection from worldview-2 data", International Journal of Computer Science and Engineering Survey, Vol.3, No.4, p.1, 2012.

[3] J. Y. Kim, L. S. Kim, and S. H. Hwang, "An advanced contrast enhancement using partially overlapped sub-block histogram equalization", IEEE Transactions on Circuits Systems for Video Technology, Vol. 11, No. 4, pp. 475-484, 2001.

[4] Y. Y. Schechner, S. G. Narasimhan, and S. K. Nayar, "Instant dehazing of images using polarization", In: Proc. of the IEEE Conference on Computer Vision and Pattern Recognition, Vol.1, pp. 325-332, 2001.

[5] S. Shwartz, E. Namer, and Y. Y. Schechner, "Blind haze separation", In: Proc. of the IEEE Conference on Computer Vision and Pattern Recognition, Vol.2, pp 1984-1991, 2006.

[6] T. Treibitz and Y. Y. Schechner, "Polarization: Beneficial for visibility enhancement?", In: Proc. of the IEEE Conference on Computer Vision and Pattern Recognition, pp. 525-532, 2009.

[7] S. G. Narasimhan and S. K. Nayar, "Chromatic framework for vision in bad weather", In: Proc. of the IEEE Conference on Computer Vision and Pattern Recognition, Vol.1, pp. 598-605, 2000.

[8] S. G. Narasimhan and S. K. Nayar, "Contrast restoration of weather degraded images", IEEE Transactions on Pattern Analysis and Machine Intelligence, Vol 25, pp.713-724, 2003.

[9] S. K. Nayar and S. G. Narasimhan, "Vision in bad weather", In: Proc. of the IEEE International Conference on Computer Vision, Vol.2, pp.820-827, 1999.

[10] C.L. Hung, Z. Ma, C.Y. Lin, and H.H. Wang, "Image Haze Removal of Optimized Contrast 
Enhancement Based on GPU", Frontier Computing, Springer, pp. 53-63, 2016.

[11] E. Alharbi, P. Ge, and H. Wang, "A Research on Single Image Dehazing Algorithms Based on Dark Channel Prior", Journal of Computer and Communications, Vol.4, pp.47-55, 2016.

[12] R. Tan, "Visibility in bad weather from a single image", In: Proc. of the IEEE Conference on Computer Vision and Pattern Recognition, pp. 1-8, 2008.

[13] P. Chavez, "An improved dark-object substraction technique for atmospheric scattering correction of multispectral data", Remote Sensing of Environment, Vol.24, pp. 450-479, 1988.

[14] M.I. Anwar and A. Khosla, "Vision enhancement through single image fog removal", Engineering Science and Technology, an International Journal, Vol. 20, No. 3, pp. 1075-1083, 2017.

[15] K. He, J. Sun, and X. Tang, "Single image haze removal using dark channel prior," IEEE Trans. Pattern Analysis and Machine Intelligence, Vol. 33, No. 12, pp. 2341-2353, 2011.

[16] L. Kratz and K. Nishino, "Factorizing scene albedo and depth from a single foggy image", In: Proc. IEEE Conference on Computer Vision, pp. 1701-1708, 2009

[17] K. Nishino, L. Kratz, and S. Lombardi, "Bayesian defogging", International Journal of Computer Vision, Vol. 98, No. 3, pp.263-278, 2012.

[18] G.F. Meng, Y. Wang, J.Y. Duan, S.M. Xiang, and C.J. Pan, "Efficient image dehazing with boundary constraint and contextual regularization", In: Proc. of the IEEE International Conference on Computer Vision, pp. 617-624, 2013.

[19] K. Tang, J. Yang, and J. Wang, "Investigating haze-relevant features in a learning framework for image dehazing", In: Proc. of the IEEE Conference on Computer Vision and Pattern Recognition, pp. 2995-3000, 2014.

[20] Q. Zhu, J. Mai, and L. Shao, "A fast single image haze removal algorithm using color attenuation prior", IEEE Transactions on Image Processing, Vol .24, No.11, pp. 3522-33, 2015.

[21] E. J. McCartney, Optics of the atmosphere: scattering by molecules and particles, New York, John Wiley and Sons, Inc. 1976.
[22] H. Koschmieder, "Theorie der horizontalen sichtweite", Beitr. Phys. Freien Atm., Vol. 12, pp.171-181, 1924.

[23] E.J. Candes and D.L. Donoho, "Curvelets, multiresolution representation, and scaling laws", SPIE Wavelet Applications in Signal and Image Processing VIII, Vol. 4119, No. 1, pp. 112, 2000.

[24] B. Eriksson, Automatic Image De-Weathering Using Curvelet-Based Vanishing Point Detection. 2011.

[25] V.M. Kamble, P. Parlewar, A.G. Keskar, and K.M. Bhurchandi, "Performance evaluation of wavelet, ridgelet, curvelet and contourlet transforms based techniques for digital image denoising", Artificial Intelligence Review, Vol.45, No.4, pp.509-533, 2016.

[26] J.L. Starck, E.J. Candès, D.L. Donoho, "The curvelet transform for image denoising", IEEE Transactions on image processing, Vol.11, No.6, pp .670-84, 2002. 